\title{
HOMOLOGY OF REGULAR COVERINGS OF SPUN CW PAIRS WITH APPLICATIONS TO KNOT THEORY ${ }^{1}$
}

\author{
W. L. MOTTER
}

\begin{abstract}
The $p$-spin of a pair of $\mathrm{CW}$ complexes, one a subcomplex of the other, is defined. The algebraic properties of certain tensor-product chain complexes are used to calculate the homology groups of regular coverings of such spun pairs where these groups are considered as modules over the integral group ring of the group of covering transformations. In $\$ 4$, by using the free differential calculus and "geometric presentations" for fundamental groups, presentations for certain homology groups are developed. In $\$ \$ 5$ and 6 these results are used to analyze the homology and associated invariants for coverings of complements of higher-dimensional knots and torus-like embeddings in the sphere obtained by $p$-spinning.
\end{abstract}

1. Introduction. Let $K$ be a connected $\mathrm{CW}$ complex and $L$ a connected subcomplex, define $\chi_{p}(K, L)$, the $p$-spin of the $C W$ pair $(K, L)$ as $S^{p} \times K$ $\cup D^{p+1} \times L$ identified along $S^{p} \times L$; here $\left(D^{p+1}, S^{p}\right)$ is the standard disk, sphere pair and $p \geqslant 1$. The process of $p$-spinning has been studied by several people [3], [5], [1], [4], [10], [2], [18], [14], [13].

If $(\tilde{K}, \Pi)$ is a regular covering of $K$ with $G$ the group of covering translations, then since $\Pi_{1}\left(\chi_{p}(K, L)\right) \cong \Pi_{1}(K)$, the associated regular covering $\tilde{\chi}_{p}(K, L)$ of $\chi_{p}(K, L)$ is $S^{p} \times \tilde{K} \cup D^{p+1} \times \tilde{L}$ identified along $S^{p} \times \tilde{L}$, where $\tilde{L}=\Pi^{-1}(L)$. Here $\gamma \in G$ acts on cells $\sigma \times \tau$ of $\tilde{\chi}_{p}(K, L)$ by $\gamma(\sigma \times \tau)$ $=\sigma \times(\gamma \tau), \sigma \subset D^{p+1}, \tau \subset \tilde{K}$ (see Figure 1).

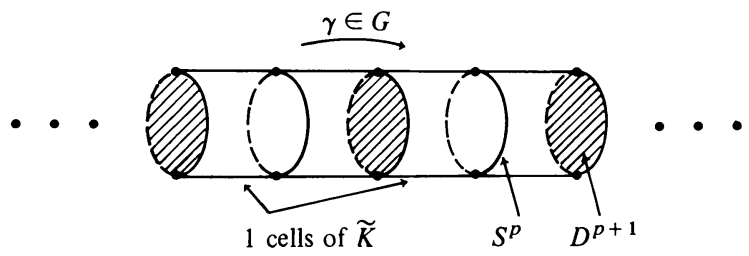

FIGURE 1

Our main result is to construct a tensor-product chain complex $C_{*}$ by which

Presented to the Society, November 24, 1972; received by the editors November 4, 1975.

AMS (MOS) subject classifications (1970). Primary 57C45, 55A10; Secondary 55A05, 55A25, $55 \mathrm{~J} 25$.

Key words and phrases. CW complexes, spun CW pairs, regular coverings, homology of product complexes, free differential calculus, presentations for homology groups, spun knots, polynomial invariants, fibered embeddings.

${ }^{1}$ Research partially supported by NSF Grant GP-19964. The major results of this paper were obtained during the author's doctoral research under the direction of D. W. Sumners at Florida State University. 
the homology of $\tilde{\chi}_{p}(K, L)$ is calculated as a module over ZG, the integral group . ring of $G$, as follows.

THEOREM 3.5.

$$
H_{i}\left(\tilde{\chi}_{p}(K, L)\right) \cong_{\mathrm{ZG}}\left\{\begin{array}{l}
H_{i}(\tilde{K}), \quad i \leqslant p, \\
H_{p+n}(\tilde{K}) \oplus H_{n}(\tilde{K}, \tilde{L}), \quad i=p+n, n \geqslant 1 .
\end{array}\right.
$$

2. Coverings of $\chi_{p}(K, L)$ and their cellular homology. Following [15], we know that the covering transformations preserve dimension, orientation, and the incidence relations of cells in $\tilde{K}$, i.e., $G$ operates on $\tilde{K}$ as a complex. Moreover, there exists a fundamental region $F$ of $\tilde{K}$ modulo $G$; namely, a subset $F$ of cells $\left\{\tilde{u}_{i}^{n}\right\}$, so that the collection $\left\{\gamma \tilde{u}_{i}^{n} \mid \tilde{u}_{i}^{n} \in F, \gamma \in G\right\}$ represents uniquely the cells of $\tilde{K}$.

The next two propositions are straightforward extensions of results from [11, pp. 187, 190] to the more general situation where the chain groups are also ZG modules.

Proposition 2.1. Let $Y$ and $X$ be $C W$ complexes with $G$ a group of transformations operating on $X$ as a complex; then the Eilenberg-Zilber transformation $T\left(\tau^{n} \otimes \sigma^{m}\right)=\tau^{n} \times \sigma^{m}$ is a natural chain equivalence of left $Z G$ modules from the tensor product complex $\left[C(Y) \otimes_{Z} C(X)\right]_{*}$ onto the chain complex $C_{*}(Y \times X) . \quad C_{*}(Y)$ is the cellular Z-chain module of $Y$; and $C_{*}(X)$ and $C_{*}(Y \times X)$ are the cellular $Z G$-chain modules of $X$ and $Y \times X$ respectively.

Proposition 2.2. If $(A, X, Y)$ are invariant under the action of $G(A$ a subcomplex of $X)$ and $F: A \rightarrow Y$ preserves the action of $G$, then the cellular $Z G$ chain complex of the adjunction complex $Y \cup_{F} X$ with $a \sim F(a)$ is chain equivalent to the $Z G$-quotient module of $C_{*}(Y) \oplus C_{*}(X)$ by $\left\{F_{\sharp}(c)-c: c \in C_{*}(A)\right\}$.

Let $i_{1}, i_{2}$ denote the inclusion maps of $S^{p} \times L$ into $S^{p} \times \tilde{K}, D^{p+1} \times \tilde{L}$ respectively. By Proposition 2.1 , we have $C_{*}\left(S^{p} \times \tilde{K}\right)$ chain equivalent to $\left[C\left(S^{p}\right) \otimes_{Z} C(\tilde{K})\right]_{*}$ and $C_{*}\left(D^{p+1} \times \tilde{L}\right)$ chain equivalent to

$$
\left[C\left(D^{p+1}\right) \otimes_{Z} C(\tilde{L})\right]_{*}
$$

under natural (Eilenberg-Zilber) transformations, say $T$ and $T^{\prime}$. Let

$$
C_{*} \equiv \frac{\left[C\left(S^{p}\right) \otimes C(\tilde{K})\right]_{*} \oplus\left[C\left(D^{p+1}\right) \otimes C(\tilde{L})\right]_{*}}{\text { Image }\left(i_{l_{\sharp}} \oplus-i_{2_{\sharp}}\right)}
$$

and $I \equiv i_{1 \sharp} \oplus-i_{2 \sharp}$ for notational convenience. With these constructions, we can now prove

THEOREM 2.3. There is a natural chain equivalence of $Z G$ modules from $C_{*}$ onto $C_{*}\left(S^{p} \times \tilde{K} \cup_{S^{p} \times \tilde{L}} D^{p+1} \times \tilde{L}\right)$.

Proof. By naturality, we have the commutative diagram: 
$0 \rightarrow C_{*}\left(S^{p} \times \widetilde{L}\right) \stackrel{I}{\longrightarrow} C_{*}\left(S^{p} \times \widetilde{K}\right) \oplus C_{*}\left(D^{p+1} \times \widetilde{L}\right) \rightarrow C_{*}\left(S^{p} \times \widetilde{K} \cup_{S^{p} \times \widetilde{L}} D^{p+1} \times \widetilde{L}\right) \rightarrow 0$

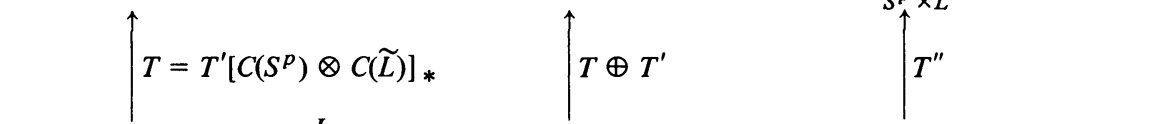

$0 \rightarrow\left[C\left(S^{p}\right) \otimes C(\widetilde{L})\right]_{*} \stackrel{I}{\longrightarrow}\left[C\left(S^{p}\right) \otimes C(\widetilde{K})\right]_{*} \oplus\left[C\left(D^{p+1}\right) \otimes C(\widetilde{L})\right]_{*} \rightarrow C_{*} \rightarrow 0$.

The top row is exact by Proposition 2.2; thus, we obtain an induced natural chain equivalence $T^{\prime \prime}$ of the quotients.

3. A formula for homology of $\tilde{\chi}_{p}(K, L)$. The structure of $C_{*}$ can be described as follows: Take $S^{p}$ as the complex consisting of a vertex $\nu$ and a $p$-cell $e^{p}$ with $\dot{e}^{p}=\nu$ and $D^{p+1}$ as $\nu \cup e^{p} \cup e^{p+1}$ with $\dot{e}^{p+1}=e^{p}$. Suppose in the covering complex $\tilde{K}$, we have vertices $g \nu$ and $n$-cells $g \tilde{u}_{1}^{n}, \ldots, g \tilde{u}_{r_{n}}^{n}$, where $g$ ranges over $G$. Denote $\tilde{u}_{i}^{n}=\tilde{l}_{i}^{n}$ if and only if $\tilde{u}_{i}^{n}$ is an $n$-cell in the subcomplex $\tilde{L}$. Thus for $m<p, C_{m}\left(C_{*}\right)$ is the free left ZG module generated by $\left\{\nu \otimes \tilde{u}_{i}^{m}\right\} ; C_{p}\left(C_{*}\right)$ is the free left ZG module generated by $\left\{e^{p} \otimes \nu\right\} \cup\{\nu$ $\left.\otimes \tilde{u}_{i}^{p}\right\}$; for $n \geqslant 1, C_{p+n}\left(C_{*}\right)$ is the free left ZG module generated by $\left\{e^{p} \otimes \tilde{u}_{i}^{n}\right\}$ $\cup\left\{e^{p+1} \otimes \tilde{l}_{i}^{n-1}\right\} \cup\left\{\nu \otimes \tilde{u}_{i}^{n+p}\right\}$. The boundary homomorphisms are given by: $\partial\left(e^{p} \otimes \tilde{u}_{i}^{n}\right)=(-1)^{p} e^{p} \otimes \partial \tilde{u}_{i}^{n}$, since $e^{p}$ is a cycle with $\partial e^{p}=0 ; \partial\left(\nu \otimes \tilde{u}_{i}^{n+p}\right)$ $=\nu \otimes \partial \tilde{u}_{i}^{n+p} ; \partial\left(e^{p+1} \otimes \tilde{l}_{i}^{n-1}\right)=e^{p} \otimes \tilde{l}_{i}^{n-1}+(-1)^{p+1} e^{p+1} \otimes \partial \tilde{l}_{i}^{n-1}$.

Using the properties of the tensor product boundary operator, the next lemma can be established.

LeMma 3.1. In $C_{p+n}\left(C_{*}\right)(n \geqslant 1)$, the cycles are linear combinations of cycles of the form $c_{i}^{*}=e^{p} \otimes \tilde{c}_{i}^{n}+(-1)^{p+1} e^{p+1} \otimes \partial \tilde{c}_{i}^{n}$, where $\tilde{c}_{i}^{n}$ is a chain in $C_{n}(\tilde{K})$ with $\partial \tilde{c}_{i}^{n} \in C_{n-1}(\tilde{L})$, or cycles of the form $\nu \otimes \tilde{z}_{i}^{n+p}$ where $\tilde{z}_{i}^{n+p}$ denotes a cycle in $C_{n+p}(\tilde{K})$.

From now on $R \equiv Z G$. Let $R\left\{c_{i}^{*}\right\}$ denote the ZG-submodule generated by $\left\{c_{i}^{*}\right\}, R\left\{\nu \otimes \tilde{z}_{i}^{p+n}\right\}$ the ZG-submodule generated by $\left\{\nu \otimes \tilde{z}_{i}^{p+n}\right\}, \bar{B}_{p+n}$ the ZGsubmodule generated by $\left\{\partial\left(e^{p} \otimes \tilde{u}_{i}^{n+1}\right)\right\} \cup\left\{\partial\left(e^{p+1} \otimes \tilde{l}_{i}^{n}\right)\right\}, B_{p+n}^{*}$ the ZG-submodule generated by $\left\{\partial\left(\nu \otimes \tilde{u}_{i}^{n+p+1}\right)\right\}$. From Lemma 3.1, it is easy to see that $H_{p+n}\left(C_{*}\right)$ has the direct sum decomposition

$$
R\left\{c_{i}^{*}\right\} / \bar{B}_{p+n} \oplus R\left\{\nu \otimes \tilde{z}_{i}^{p+n}\right\} / B_{p+n}^{*} .
$$

Clearly,

$$
R\left\{\nu \otimes \tilde{z}_{i}^{n+p}\right\} / B_{p+n}^{*} \cong_{R} H_{p+n}(\tilde{K}) .
$$

LEMMA 3.2. $H_{p+n}\left(C_{*}\right) \cong_{R} H_{p+n}(\tilde{K}) \oplus H_{n}(\tilde{K}, \tilde{L}), n \geqslant 1$.

Proof. From the above, it must be shown that $R\left\{c_{i}^{*}\right\} / \bar{B}_{p+n} \cong_{R} H_{n}(\tilde{K}, \tilde{L})$. Now from Lemma 3.1, $c_{i}^{*}$ can be represented by

$$
\left(e^{p} \otimes \sum a_{i} \tilde{u}_{i}^{n}+(-1)^{p+1} e^{p+1} \otimes \partial\left(\sum a_{i} \tilde{u}_{i}^{n}\right)\right)
$$

where $\tilde{c}_{i}^{n}=\sum a_{i} \tilde{u}_{i}^{n}$ is an element of ker $\bar{\partial}$; so $\Psi\left(c_{i}^{*}\right)=\left\langle\tilde{c}_{i}^{n}\right\rangle$ gives a ZGhomomorphism from $R\left\{c_{i}^{*}\right\}$ onto $H_{n}(\tilde{K}, \tilde{L})=\operatorname{ker} \bar{\partial} / B_{n}(\tilde{K}, \tilde{L})$. Here \langle\rangle is an equivalence class in $\operatorname{ker} \bar{\partial} / B_{n}(\tilde{K}, \tilde{L})$, where $\bar{\partial}=p \circ \partial$ with $p: C_{n-1}(\tilde{K})$ 
$\rightarrow C_{n-1}(\tilde{K}) / C_{n-1}(\tilde{L})$ the canonical projection and $\partial: C_{n}(\tilde{K}) \rightarrow C_{n-1}(\tilde{K})$. Finally, $\left\langle\tilde{c}_{i}^{n}\right\rangle=0$ if and only if $\sum a_{i} \tilde{u}_{i}^{n}=\partial\left(\sum s_{i} \tilde{u}_{i}^{n+1}\right)-\sum b_{i} \tilde{l}_{i}^{n}$, in which case $\partial\left(\sum a_{i} \tilde{u}_{i}^{n}\right)=-\partial\left(\sum b_{i} \tilde{l}_{i}^{n}\right)$, i.e., the preimage

$$
c_{i}^{*}=(-1)^{p+2} \partial\left(e^{p} \otimes \sum s_{i} \tilde{u}_{i}^{n+1}\right)-\partial\left(e^{p+1} \otimes \sum b_{i} \tilde{l}_{i}^{n}\right) \in \bar{B}_{p+n} ;
$$

hence $\bar{B}_{p+n}=$ kernel of $\Psi$.

REMARK 3.3. Geometrically, relative $n$-cycles of $C_{n}(\tilde{K}, \tilde{L})$ "spin" to $(p+n)$ cycles of $C_{p+n}\left(\tilde{\chi}_{p}(K, L)\right)$.

Proposition 3.4. For $r \leqslant p, H_{r}\left(C_{*}\right) \cong H_{r}(\tilde{K})$.

Proof. For $r<p$, the truncated chain complex $\left\{C_{r}\left(C_{*}\right), \partial_{r}\right\}$ is chain isomorphic to $\left\{C_{r}(K), \partial_{r}\right\}$ by $\nu \otimes \tilde{u}_{j}^{r} \leftrightarrow \tilde{u}_{j}^{r}$; moreover, since $\partial\left(e^{p+1} \otimes \nu\right)=e^{p}$ $\otimes \nu, H_{p}\left(C_{*}\right) \cong_{\mathrm{ZG}} H_{p}(\tilde{K})$.

Lemma 3.2 and Proposition 3.4 establish Theorem 3.5.

4. Presentations for $p+1$ homology of the universal covering $\tilde{\chi}_{p}(K, L)$. By shrinking to a point a suitable maximal tree of $(K, L)$, we may reduce our considerations to pairs $(K, L)$ having only one vertex. Suppose $\Pi_{1}(K)$ has a "geometric presentation", $\left|x_{0}, \ldots, x_{n}: r_{1}, \ldots, r_{m}\right|$ where $x_{0}, \ldots, x_{r}$ are generators for $\Pi_{1}(L)$. The 2-skeleton of $K$ consists in one vertex $\nu, n+1$ edges $\varepsilon_{0}, \varepsilon_{1}, \ldots, \varepsilon_{n}$ and $m$ 2-cells $p_{1}, \ldots, p_{m}$, where $x_{j}$ is carried by $\varepsilon_{j}$ and $r_{j}$ by $p_{j}$. In the 2-skeleton of $\tilde{K}$, the universal cover of $K$, we have 0 -cells $g \nu$; 1-cells $g \tilde{\varepsilon}_{0}, \ldots, g \tilde{\varepsilon}_{n} ; 2$-cells $g \tilde{p}_{1}, \ldots, g \tilde{p}_{m}$; where $g$ ranges over $G \cong \Pi_{1}(K)$. Using the same reasoning as $[10, \S 2]$, we know the boundary homomorphisms of

$$
\cdots \rightarrow C_{2}(\tilde{K}) \stackrel{\partial_{2}}{\longrightarrow} C_{1}(\tilde{K}) \stackrel{\partial_{1}}{\longrightarrow} C_{0}(\tilde{K}) \rightarrow 0
$$

are defined by

$$
\partial_{1}\left(g \tilde{\varepsilon}_{j}\right)=g\left(\partial_{1} \tilde{\varepsilon}_{j}\right)=g\left(x_{j}-1\right) \nu, \quad \partial_{2}\left(g \tilde{p}_{i}\right)=g\left(\sum_{j=0}^{n} \frac{\partial r_{i}}{\partial x_{j}} \tilde{\varepsilon}_{j}\right),
$$

where $\partial r_{i} / \partial x_{j}$ are the Fox Free Derivatives.

Let $\bar{C}_{*}$ be the chain subcomplex of $C_{*}$ generated by $\left\{e^{p} \otimes \tilde{u}_{i}^{n}\right\} \cup\left\{e^{p+1}\right.$ $\left.\otimes \tilde{l}_{i}^{n-1}\right\}$. As left $Z \Pi_{1}(K)$ modules, Lemma 3.2 gives for any $n \geqslant 1, H_{n}(\tilde{K}, \tilde{L})$ $\cong H_{p+n}\left(\bar{C}_{*}\right)$ so that

$$
H_{p+n}\left(\tilde{\chi}_{p}(K, L)\right) \cong H_{p+n}\left(C_{*}\right) \cong H_{p+n}(\tilde{K}) \oplus H_{n}\left(\bar{C}_{*}\right) .
$$

To compute $H_{p+1}\left(\bar{C}_{*}\right)$, we observe by Lemma 3.1 that here the $(p+1)$-cycles of $\bar{C}_{*}$ are generated by $z_{i}=e^{p} \otimes \tilde{\varepsilon}_{i}+(-1)^{p+1}\left(e^{p+1} \otimes \partial \tilde{\varepsilon}_{i}\right), i=0, \ldots, n$, since $\partial \tilde{\varepsilon}_{i}=\left(x_{i}-1\right) \nu \in \tilde{L}$ for all $i$. In fact, it is easy to show that $\left\{z_{i}\right\}$ freely generates the submodule of cycles of $\bar{C}_{*}, \bar{Z}_{p+1}\left(\bar{C}_{*}\right)$, as a $Z \Pi_{1}(K)$ module. We can now prove

THEOREM 4.1. If $\Pi_{1}(K)$ has a presentation $\left|x_{0}, \ldots, x_{n}: r_{1}, \ldots, r_{m}\right|$ with $x_{0}, \ldots, x_{r}$ the image of the generators of $\Pi_{1}(L)$ under the inclusion map, then $\left|z_{r+1}, \ldots, z_{n}: \sum\left(\partial r_{i} / \partial x_{j}\right) z_{j}(i=1, \ldots, n)\right|$ is a presentation for $H_{1}(\tilde{K}, \tilde{L})$ $\cong H_{p+1}\left(\bar{C}_{*}\right)$ as a left $Z \Pi_{1}(K)$ module. 
Proof. $C_{p+2}\left(\bar{C}_{*}\right)$ is the free left $Z \Pi_{1}(K)$ module generated by $\left\{e^{p} \otimes \tilde{p}_{j}\right\}$ $\cup\left\{e^{p+1} \otimes \tilde{\varepsilon}_{0}, \ldots, e^{p+1} \otimes \tilde{\varepsilon}_{r}\right\}$. From the algebra of the tensor product complex $C_{*}$, we observe that

$$
\partial\left(e^{p} \otimes \tilde{p}_{i}\right)=(-1)^{p} \sum \frac{\partial r_{i}}{\partial x_{j}}\left(e^{p} \otimes \tilde{\varepsilon}_{j}\right)=(-1)^{p} \sum \frac{\partial r_{i}}{\partial x_{j}} z_{j},
$$

since $\sum\left(\partial r_{i} / \partial x_{j}\right)\left(x_{i}-1\right) \nu=\partial_{1}\left(\partial_{2} \tilde{p}_{i}\right)=0$. Moreover, $\partial\left(e^{p+1} \otimes \tilde{\varepsilon}_{0}\right)=z_{0}$, $\ldots, \partial\left(e^{p+1} \otimes \tilde{\varepsilon}_{r}\right)=z_{r}$. This establishes the theorem for one particular presentation of $\Pi_{1}(K)$. The method of [10, p. 97] establishes it in general.

5. Applications to coverings of complements of spun knots. We obtain a method (Theorem 5.2) of calculating the homotopy groups of spun knots as left $Z \Pi_{1}$ modules and a method (Theorem 5.4) of proving that the polynomial invariants of the knot one is spinning completely determine the invariants of the spun knot. An $n$-knot denotes a smooth embedding $\kappa: S^{n} \rightarrow S^{n+2}$. For a definition of the process of $p$-spinning $\kappa$ to obtain an $(n+p)$-knot and the notation of this section, see [7, p. 415]. Call this spun knot $\chi_{p}(\kappa)\left(S^{n}\right)$. For a proof of the next lemma, see [7, p. 416].

Lemma 5.1. If $Y=S^{n+p+2}-\chi_{p}(\kappa)\left(S^{n}\right)$ then $Y \simeq \chi_{p}(K, L)$, where $K$ $\simeq S^{n+2}-\kappa\left(S^{n}\right)$ and $L \simeq S^{n+1}-S^{n-1}$, a homotopy circle, represents a meridian in $\Pi_{1}(K)$. $\simeq$ denotes homotopy equivalence.

THeOREM 5.2. Let $K, L$, and $Y$ be as in Lemma 5.1, $\sim$ denote universal cover, and $G=\Pi_{1}(K)=\left|x_{0}, x_{1}, \ldots, x_{n}: r_{1}, \ldots, r_{m}\right|$, with $L$ the carrier of $x_{0} ;$ then

$$
H_{i}(\tilde{Y}) \cong_{\mathrm{ZG}} \begin{cases}H_{i}(\tilde{K}), & i \leqslant p, \\ H_{n+p}(\tilde{K}) \oplus H_{n}(\tilde{K}), & i=n+p, n>1, \\ H_{p+1}(\tilde{K}) \oplus H_{1}(\tilde{K}, \tilde{L}), & i=p+1 .\end{cases}
$$

(2) $H_{1}(\tilde{K}, \tilde{L})$ is presented by $\left|z_{1}, \ldots, z_{n}: \sum \partial r_{i} / \partial x_{j}(i=1, \ldots, n)\right|$.

Proof. (1) follows directly from Theorem 3.5 and the exact sequence for homology of the pair $(\tilde{K}, \tilde{L})$ since $L$ is a 1 -sphere with $H_{r}(\tilde{L})=0$ when $r \geqslant 1$. Observe that $H_{1}(\tilde{K}, \tilde{L}) \cong \operatorname{ker} H_{0}(\tilde{L}) \rightarrow H_{0}(\tilde{K})$. (2) follows from Theorem 4.1.

See [7, pp. 416-417] for a completely different approach for obtaining group presentations for $\operatorname{ker} H_{0}(\tilde{L}) \rightarrow H_{0}(\tilde{K})$.

COROLlaRY 5.3. If $H_{i}(\tilde{K})=0, i \leqslant p+1$, as in the case of the p-spin of a 1knot $\left(S^{3}, \kappa\left(S^{1}\right)\right)$, then

$$
H_{1}(\tilde{K}, \tilde{L}) \cong_{Z \Pi_{1}} H_{p+1}(\tilde{Y}) \cong_{Z \Pi_{1}} \Pi_{p+1}(Y)
$$

and $\Pi_{p+1}(Y)$ is presented as in (2).

Compare this with [1] and [10]. The same type of result for the $p+1$ homotopy groups of spun links is proved by purely geometric constructions in [13].

Let $\Lambda(\Gamma)$ denote the integral group rings of the $\infty$-cyclic groups $Z(t)(Q(t))$ respectively. The polynomial invariants of an $n$-knot are invariants of $\Lambda(\Gamma)$ - 
structure of $H_{*}(\tilde{K} ; Z)$, where $\tilde{K}$ is the $\infty$-cyclic cover of the knot complement $K \simeq S^{n+2}-\kappa\left(S^{n}\right)$. See [9] and [16]. We now prove

THEOREM 5.4. Let $K, L$, and $Y$ be as in Lemma 5.1; then the $\infty$-cyclic covering $\tilde{Y} \simeq \tilde{\chi}_{p}(K, L)$ has homology $[Z(Q)$ coefficients $]$ :

$$
H_{i}(\tilde{Y}) \cong_{\Lambda(\Gamma)} \begin{cases}H_{i}(\tilde{K}), & i \leqslant p \\ H_{n+p}(\tilde{K}) \oplus H_{n}(\tilde{K}), & i=n+p, n \geqslant 1 .\end{cases}
$$

Proof. The proof is a direct application of Theorem 3.5 and the facts that as in Theorem 5.2 for $n>1, H_{n}(\tilde{K}, \tilde{L}) \cong H_{n}(K)$; also $H_{1}(\tilde{K}, \tilde{L}) \cong H_{1}(\tilde{K})$ as ker $H_{0}(\tilde{L}) \stackrel{i *}{\longrightarrow} H_{0}(\tilde{K})$ here is 0 since for $\infty$-cyclic covering $H_{0}(\tilde{L}) \cong C_{0}(\tilde{\mathcal{v}})$ $\cong H_{0}(\tilde{K})$.

6. Torus-like embeddings obtained by spinning and their invariants. Let $\kappa$ be a compact, connected, orientable, codimension 2, smooth submanifold contained in the interior of $B^{n}$, the closed unit $n$-ball. By $q$-spinning the pair $\left(B^{n}, \kappa\right)$, we obtain a sphere $S^{q+n}=S^{q} \times B^{n} \cup D^{q+1} \times \partial B^{n}$ identified along $S^{q} \times \partial B^{n}$ and a spun torus-like smooth submanifold $M=S^{q} \times \kappa \subset S^{q+n} . M$ has trivial normal disk bundle $N \approx M \times D^{2}$ (see [12]); so by Alexander duality, the complement $S^{q+n}-\left(S^{q} \times \kappa\right)=Y \simeq S^{n}-\stackrel{N}{\text { has }} H_{1}(Y)=Z(t)$, the infinite cyclic group generated by $t$. The polynomial invariants of the homology of the $\infty$-cyclic covering complex $\tilde{Y}$ of $Y$ can then be defined as with knot complements (see [16, §2] and [14, Chapter 2]). This section shows these are also completely determined by the invariants of $S^{n}-\kappa$.

Using the result that $B^{n}-\kappa \simeq\left(S^{n}-\kappa\right) \vee_{*} S^{n-1}$, where $S^{n-1}=\partial B^{n}$ (see [16, p. 111]), it can be proved (see [14, pp. 1-3]) that $Y$ is homotopy equivalent to

$$
S^{q} \times\left(S^{n}-\kappa\right) \underset{S^{q} \times\{*\}}{\cup} D^{q+1} \times\{*\} \vee_{*} S^{n-1} \equiv X \vee_{*} S^{n-1}
$$

and $\tilde{Y} \simeq \tilde{X} \cup_{\{*\}}\left(\cup_{i \in Z} S_{i}^{n-1}\right)$. Here $\{\tilde{*}\}=\pi^{-1}(*)$, where $\pi:\left(\widetilde{\left.S^{n}-\kappa\right)} \rightarrow\left(S^{n}\right.\right.$ $-\kappa)$ is the covering projection. The action of $Z(t)$ on $\tilde{Y}$ preserves not only the above subspace splitting for $\tilde{Y}$, but also the product structure of each of the pieces $S^{q} \times\left(\widetilde{S^{n}-\kappa}\right), D^{q+1} \times\{\tilde{*}\}$, and $S^{q} \times\{\tilde{*}\}$. Thus, by Mayer-Vietoris

$$
\cdots \rightarrow H_{r}(\{\tilde{*}\}) \rightarrow H_{r}(\tilde{X}) \oplus H_{r}\left(\bigcup_{i \in Z} S_{i}^{n-1}\right) \rightarrow H_{r}(\tilde{Y}) \rightarrow \cdots
$$

is an exact sequence of $\Lambda$-modules. Finally, since $H_{r}(\{\tilde{*}\})=0, r$ $\neq 0$ and $H_{0}(\{\tilde{*}\}) \cong H_{0}\left(D^{q+1} \times\{\tilde{*}\}\right)$ and $H_{0}(\{\tilde{*}\}) \cong H_{0}\left(\cup_{i \in Z} S_{i}^{r-1}\right)$, we have by exactness the following.

THEOREM 6.1. $H_{r}(\tilde{Y}) \cong_{\Lambda} H_{r}(\tilde{X}) \oplus H_{r}\left(\cup_{i \in Z} S_{i}^{n-1}\right), r \geqslant 1$.

Since

$$
H_{r}\left(\bigcup_{i \in Z} S_{i}^{n-1}\right)= \begin{cases}\Lambda, & r=n-1, \\ 0, & \text { otherwise }\end{cases}
$$

to compute homology of $\tilde{Y}$ we can use the following formula for the homology of $\tilde{X}$. 


\section{THEOREM 6.2.}

$$
H_{r}(\tilde{X}) \cong \begin{cases}H_{r}\left(\widetilde{S^{n}-\kappa}\right), & r \leqslant q, \\ H_{q+1}\left(\widetilde{S^{n}-\kappa}\right) \oplus H_{1}\left(\widetilde{S^{n}-\kappa}\right) \oplus \Lambda, & r=q+1, \\ H_{q+n}\left(\widetilde{S^{n}-\kappa}\right) \oplus H_{n}\left(\widetilde{S^{n}-\kappa}\right), & r=q+n, n \geqslant 2 .\end{cases}
$$

Proof. This is a direct result of Theorem 3.5 with $K=\left(S^{n}-\kappa\right), L=\{*\}$ $=\nu$, and $G=Z(t)\left(X=\chi_{q}(K, L)\right)$ and the following facts. The exact homology sequence of the pair $\left(\widetilde{S^{n}-\kappa},\{*\}\right)$ gives $H_{n}\left(\widetilde{S^{n}-\kappa},\{\tilde{*}\}\right) \cong H_{n}\left(\widetilde{S^{n}-\kappa}\right)$ for $n \geqslant 2$. When $n=1$,

$$
0 \rightarrow H_{1}\left(\widetilde{S^{n}-\kappa}\right) \rightarrow H_{1}\left(\widetilde{S^{n}-\kappa},\{\tilde{*}\}\right) \rightarrow H_{0}^{\sharp}(\{\tilde{*}\}) \rightarrow 0
$$

is exact. Claim $H_{0}^{\sharp}(\{\tilde{*}\}) \cong \Lambda$ since $\Lambda \cong C_{0}(\{\tilde{*}\})$ and if $\varepsilon(t)=1$, we have the exact sequence

$$
0 \rightarrow \Lambda \stackrel{(t-1)}{\longrightarrow} \Lambda \stackrel{\varepsilon}{\rightarrow} Z \rightarrow 0 .
$$

So (1) splits, and $H_{1}\left(\widetilde{S^{n}-\kappa},\{\tilde{*}\}\right) \cong_{\Lambda} H_{1}\left(\widetilde{S^{n}-\kappa}\right) \oplus \Lambda$. Note that $\Lambda$ can be replaced by $\Gamma$ in the preceding.

Corollary 6.3. Fibered embeddings of the form $S^{q} \times \kappa \subset S^{q+n}$ cannot be obtained by q-spinning the pair $\left(B^{n}, \kappa\right)$ since for a fibered embedding, the $\infty$-cyclic cover of the complement must have all homology groups finitely generated Zmodules (see $[2, p .416])$ and $\Lambda$ is not a finitely generated Z-module.

\section{REFERENCES}

1. J. J. Andrews and S. J. Lomonaco, Jr., The second homotopy group of spun 2-spheres in 4space, Ann. of Math. (2) 90 (1969), 199-204. MR 40 \#3547.

2. J. J. Andrews and D. W. Sumners, On higher-dimensional fibered knots, Trans. Amer. Math. Soc. 153 (1971), 415-426. MR 42 \#6808.

3. E. Artin, Zur Isotopic zweidimensionaler Fläachen in $R_{4}^{*}$, Abh. Math. Sem. Univ. Hamburg 4 (1926), 174-177.

4. S. Cappel, Superspinning and knot complements, Topology of Manifolds (Proc. Inst. Univ. of Georgia, Athens, Ga., 1969), Markham, Chicago, Ill., 1970, pp. 358-383. MR 43 \#2711.

5. D. B. A. Epstein, Linking spheres, Proc. Cambridge Philos. Soc. 56 (1960), 215-219. MR 22 $\# 8514$.

6. R. H. Fox, Free differential calculus. I. Derivation in the group ring, Ann. of Math. (2) 57 (1953), 547-560; II. The isomorphism problem of groups, Ann. of Math. (2) 59 (1954), 196-210; III. Subgroups, Ann. of Math. (2) 64 (1956), 407-414. MR 14, 843; 15, 931; 20 \#2374.

7. C. Mc A. Gordon, Some higher-dimensional knots with the same homotopy groups, Quart. J. Math. Oxford Ser. (2) 24 (1973), 411-422. MR 48 \# 5089.

8. M. Kervaire, Les noeds de dimensions supérieures, Bull. Soc. Math. France 93 (1965), 225-271. MR 32 \#6479.

9. Jerome Levine, Polynomial invariants of knots of codimension two, Ann. of Math. (2) 84 (1966), 537-554. MR 34 \#808.

10. S. J. Lomonaco, Jr., The second homotopy group of a spun knot, Topology 8 (1969), 95-98. MR 38 \#6594.

11. A. T. Lundell and S. Weingram, Topology of $C W$ complexes, Van Nostrand Reinhold, New York, 1969.

12. W. S. Massey, On the normal bundle of a sphere imbedded in Euclidean space, Proc. Amer. Math. Soc. 10 (1959), 959-964. MR 22 \#237. 
13. W. A. McCallum, The higher homotopy groups of $k$-spun knots and links, Ph.D. Thesis, Florida State Univ., Tallahassee, Fla., 1973.

14. W. L. Motter, Smooth embeddings of $S^{p} \times S^{q}$ in $S^{p+q+2}$, Ph.D. Thesis, Florida State Univ., Tallahassee. Fla., 1973.

15. K. Reidemeister, Complexes and homotopy chains, Bull. Amer. Math. Soc. 56 (1950), 297-307. MR 12, 120.

16. Y. Shionohara and D. W. Sumners, Homology invariants of cyclic coverings with applications to links, Trans. Amer. Math. Soc. 163 (1972), 101-121.

17. E. H. Spanier, Algebriac topology, McGraw-Hill, New York, 1966. MR 35 \# 1007.

18. D. W. Sumners, The effect of spinning and twist-spinning on some knot invariants, Florida State University (preprint).

Department of Mathematics, Florida A \& M University, Tallahassee, Florida 32307 